\title{
Portal Biliopathy: A Review of Imaging Features of Nine Patients
}

\author{
Ali Nawaz Khan ${ }^{1 *}$, Ken Uzoka1, Sumaira Macdonald ${ }^{2}$, Abeeku Afedzi Hammond ${ }^{3}$, \\ Anthony Kodzo-Grey Venyo ${ }^{4}$
}

${ }^{1}$ North Manchester General Hospital, Manchester, UK

${ }^{2}$ Silk Road Medical, Inc., Sunnyvale, CA, USA

${ }^{3}$ Department of Trauma and Orthopaedics, Royal Oldham Hospital, Pennine Acute Hospitals NHS Trust, Bury, UK

${ }^{4}$ Department of Urology Delaunay, North Manchester General Hospital, Manchester, UK

Email: ^drkhan1966@msn.com, kuzoka@gmial.com, sumairamacdonald@gmail.com, hammonda04@yahoo.com,

akodzogrey@yahoo.co.uk

How to cite this paper: Khan, A.N., Uzoka, K., Macdonald, S., Hammond, A.A. and Venyo, A.K.-G. (2017) Portal Biliopathy: A Review of Imaging Features of Nine Patients. International Journal of Clinical Medicine, 8, 604-617.

https://doi.org/10.4236/ijcm.2017.811057

Received: August 23, 2017

Accepted: November 21, 2017

Published: November 24, 2017

Copyright (c) 2017 by authors and Scientific Research Publishing Inc. This work is licensed under the Creative Commons Attribution International License (CC BY 4.0).

http://creativecommons.org/licenses/by/4.0/

(c) (i) Open Access

\begin{abstract}
Aim: To discuss the etiology, pathogenesis, diagnosis and management of PB, supported by a series of 9 cases from a personal series. Methods: Radiological images of 9 cases of $\mathrm{PB}$ were retrieved from different hospitals and studied and information relating to $\mathrm{PB}$ was obtained from various internet databases including PUB Med, Google, Google Scholar and Educus. Results: Portal biliopathy (PB) is a rare complication of extra-portal venous obstruction. Most patients remain asymptomatic, but some may present with raised alkaline phosphatase level, abdominal pain, fever, and cholangitis. It tends to be associated with gallbladder (GB) wall abnormalities, a cavernous transformation of the portal vein and choledochal varices. Extrinsic compression of the common bile duct, (CBD), by dilated venous collaterals together with pericholedochal fibrosis from the inflammatory process can cause portal thrombosis, which may lead to biliary stricture and dilatation of the proximal biliary tree. These strictures set the scene for formation biliary stones and cholangitis. Liver function test abnormalities are common but remain non-specific. Imaging features are instrumental in the diagnosis of PB. This paper presents 9 of such cases of portal biliopathy, with discussions on their causation, the course of the disease process and management particularly minimally invasive procedure. The role of ultrasound (US), computed tomography (CT), magnetic resonance imaging (MRI), endoscopic retrograde cholangiopancreatography (ERCP) and direct puncture cholangiography is discussed. Surgical management is evaluated. An imaging atlas is provided. Conclusions: $\mathrm{PB}$ is a rare but significant complication of portal venous obstruction that has an insidious onset and may remain
\end{abstract}


asymptomatic until late. PB has associated coagulopathies, and a variety of other disorders, which are discussed, illustrated and the diagnosis-elaborated. The role of MRI as a non-invasive imaging tool is emphasized.

\section{Keywords}

Portal Biliopathy, Portal Hypertension, Portal Venous Thrombosis, Cavernous Transformation, Biliary Strictures Imaging Portal Biliopathy

\section{Introduction}

PB is characterized by bile duct and GB wall abnormalities associated with portal hypertension (PH). PB is often associated with idiopathic extrahepatic portal venous occlusion [1] [2]. The abnormalities recorded in PB are bile duct compression, stenoses, and strictures interspaced by dilated ducts both intrahepatic and extrahepatic are affected. GB varices may present as a thickened wall, elegantly demonstrated by Doppler interrogation. PB may mimic a cholangiocarcinoma, sclerosing cholangitis, or bile duct calculi.

The association of jaundice and common bile duct compression and $\mathrm{PH}$ were first described by Fraser et al. [3] in 1944. The term "portal biliopathy" was coined in the early 1990s [1].

Accurate diagnosis is achieved by using appropriate imaging, which includes ERCP, PTC, CT and MRI/MRCP. Presently there is no role for intravenous cholangiography. Shin SM in their study of MRI features of PB showed that MR cholangiography and 3D gradient-echo imaging not only detect portal vein occlusion, but cavernous transformation, and GB varices and other associated bile duct abnormalities [4]-[11].

\section{Summary of Cases Experienced}

The aim of this study has been to present a series of cases evaluated, treated and followed at a tertiary care public institution. Patients with PB were exposed to different therapeutic approaches focused on the management of portal hypertension and biliary decompression. They were followed for $\sim 5$ years. Three cases achieved a favorable outcome with symptom remission, but one patient died while attempting dilatation of the bile duct another died because of multiple underlying pathologies. Finally, we carried out a literature review of portal hypertensive biliopathy therapeutics. Presently there is no consensus on the optimal treatment PB. The ultimate goal decompression of the biliary tree should be individually evaluated to choose the best treatment option. The literature was reviewed supported by data from our series, with a view to offering the best possible option for the management of this rare complication of PVT. The liver function abnormalities in PB are highly non-specific, and only imaging provides guidance for the most appropriate management. Imaging feature in 9 cases illustrates the 
later statement. What the imaging features from our case series is discussed individually in the images (please see images of the nine cases).

\section{Discussion and Literature Review}

$\mathrm{PB}$ is associated with $\mathrm{PH}$, particularly in patients with extrahepatic portal vein obstruction. The signs of PB include bile duct and GB abnormalities. Portal cavernoma formation, choledochal varices and ischemic injury of the bile duct have been implicated in the causation of PB. The majority of the patients with $\mathrm{PB}$ remain asymptomatic while a minority have a raised alkaline phosphatase, abdominal pain, fever, and cholangitis. Choledocholithiasis is a known complication, causing obstructive jaundice with or without cholangitis. Endoscopic sphincterotomy and stone extraction can alleviate cholangitis and jaundice associated CBD calculi. Definitive treatment by decompressive shunt surgery is sometimes required with progressive disease [2].

Several mechanisms are involved in the genesis of incomplete PB. The mechanisms include induction of fibrosis in the biliary tract related either directly to dilated peribiliary portal venous collaterals and recurrent cholangitis, loss of bile duct motility and chronic cholestasis secondary to fibrosis or bile calculi. The management of biliary calculi in patients with portal vein cavernous transformation is complex. Moreover, a heterogeneous clinical presentation and concomitant pathophysiological changes that take place in biliary anatomy, diagnosis and therapy can become very complicated. Conventional treatment of cholelithiasis, such as a sphincterotomy and balloon dilatation of bile ducts can cause complications such as cholangitis, biliary strictures, and hemobilia. Harmanci \& Bayraktar reviewed the management bile calculi related to bile duct obstruction in non-cirrhotic portal vein thrombosis and present evidence in the current literature [12].

Rarely PB is only diagnosed at laparotomy. Colle et al. [13] reported a man, 51-year of age with a history of PVT that presented with elevated liver enzymes and mass at the liver hilum. CT and MRI confirmed a hilar mass and compression of the CBD. ERCP showed irregular narrowing of the mid-CBD. The patient underwent an explorative laparotomy, which revealed a hypervascular mass in the porta hepatis surrounded by dilated by portal vein tributaries. The diagnosis of portal cavernoma was made. Further hematological tests showed an anti-phospholipid syndrome and myeloproliferative disorder as a cause of PVT. The patient was started on oral anticoagulants. In conclusion, the authors reported a patient with a biliary stricture secondary to a portal venous thrombosis and a cavernoma (PB), not diagnosed preoperatively [13].

$\mathrm{PH}$ and $\mathrm{PT}$ is a rare cause of $\mathrm{PB}$ in children with extrahepatic portal vein obstruction with $\mathrm{PH}$. Children may be asymptomatic or could lead to cholestatic liver biochemistry. El-Matary et al. reported a child that developed a cholestatic picture with $\mathrm{PH}$ some, time after having neonatal surgery for duodenal atresia. Symptomatic PB is rare in children but should be suspected in children patients 
with $\mathrm{PH}$ and features of bile duct obstruction. If endoscopic or percutaneous biliary intervention is unsuccessful, and there is no response to portal-systemic shunt, hepaticojejunostomy may have a therapeutic role in selected children [14].

$\mathrm{PB}$ is predominantly related to a combination of $\mathrm{PH}$ and extra-hepatic portal vein thrombosis. A rare case of partial intrahepatic venous thrombosis associated with acute pancreatitis, probably due to kissing gastric ulcers perforation has been described. The patient developed a portal cavernoma in addition to partial PV thrombosis [15]. A literature search showed a case where multiple duodenal ulcers in hypertensive portal duodenopathy associated with upper GI bleeding and cirrhosis was described [16].

Imaging of the bile ducts is the most important consideration in the diagnosis of PB. Arrivé et al. [7] studied and described several limitations of MRI of cholangitis. The authors suggest a systematic approach, which should include evaluation of bile duct stenoses, duct dilatation, intrahepatic calculi and inhomogeneous liver parenchyma and $\mathrm{PH}$ enhancement of the liver parenchyma and any enhancement and bile duct walls.

Ozkavukcu and associates [8] studied 16 patients to evaluate the frequency MRCP findings in PB. Described in the order of frequency was as follows: biliary stenosis, a wavy appearance of the bile ducts, angulation of the CBD, and upstream dilatation of the bile ducts.

Chevallier et al. [4] studied 10 patients with $\mathrm{PB}$ in a retrospective and monocentric study and concluded that MRC constituted an accurate imaging modality to investigate noninvasively patients with $\mathrm{PB}$.

Aguilar-Olivos et al. [9] studied 4 patients with PB from a tertiary referral center and suggested that $\mathrm{PB}$ is an underdiagnosed condition because only some patients have symptoms.

MRI has certain limitations. Matsuo et al. [10] reported a pseudo PV thrombosis on MRI in a 65-year-old woman with severe cirrhosis. Unenhanced T2-weighted fast spin-echo images showed an area of high signal intensity occupying the left second-order portal vein branch, suggesting portal vein thrombosis in cirrhosis. Doppler sonography, showed no flow in the involved portal vein, supporting the diagnosis. Gadolinium-enhanced MRI subsequently revealed the patency of the vessel. Matsuo et al. [10] concluded that an extremely slow portal venous flow might thus cause a false-positive finding venous thrombosis with unenhanced MRI and Doppler sonography.

Nunoi $\mathrm{H}$ et al. [11] described a patient with $\mathrm{PB}$-associated with the morphological abnormality of the biliary duct and GB associated with PH that presented with essential thrombocythemia, initially diagnosed with extrahepatic portal vein obstruction. The contrast-enhanced CT findings were similar to those of cholangiocarcinoma or sclerosing cholangitis. However, color Doppler and contrast-enhanced ultrasound (US) were more precise, leading to a diagnosis $\mathrm{PB}$, which prevented the patient, resulting in the avoidance of risky procedures such as bile duct biopsies. 


\section{Management}

Concerning management, Khan et al. [17] made the ensuing iterations:

The vast majority of patients with PB are usually asymptomatic and found incidentally, but rarely the patients present with abdominal pain, jaundice, weight loss and pyrexia; Treatment is instituted in symptomatic patients only, modified by the clinical picture and the stage of the disease process; The presence of severe $\mathrm{PH}$, endoscopic biliary intervention is usually the first line of treatment, considered safe and often sufficient; in patients that fail to respond to endoscopic intervention, surgery may be considered; When surgery is indicated, a portosystemic shunt prior to biliary bypass provides early symptomatic relief and may preclude biliary bypass surgery.

\section{Portal-Systemic Shunt}

Chaudhary et al. [18] retrospectively reviewed the surgical management of symptomatic PB in 9 patients. Eight of the 9 patients had jaundice, two presented with abdominal pain and one with recurrent cholangitis. Bile duct strictures and bile duct abnormalities were found in 8 patients, and two patients had choledocholithiasis on Endoscopic retrograde cholangiography (ERCP). Placement of a Portal-systemic shunt relieved jaundice in five of seven patients, and in two patients required a second-stage hepatico-jejunostomy. The authors suggested that a direct approach to the biliary tract without a preliminary shunt may be hazardous and is frequently not necessary.

\section{Failed Splenorenal Shunt and Hepaticojejunostomy}

Vleggaar and associates [19] described their experience in four adult patients with portal vein thrombosis that presented with symptoms or signs of biliary obstruction. Cholangiography revealed smooth extrinsic indentations CBD. The patients had a varied clinical course from spontaneous resolution and returned to normal liver function tests in two patients. Two patients had persistent cholestasis and required, surgical treatment failed due to the presence of numerous collateral veins.

\section{The Percutaneous Trans-Hepatic Approach, Allow Stone Extraction and Balloon Dilatation of the Bile Duct Strictures}

Perego and associates [20] describe a case of obstructive jaundice and cholangitis secondary to calculi within the $\mathrm{CBD}$ associated with bile duct strictures after a cavernous transformation of the portal vein. Biliodigestive anastomosis was not appropriate as the patient suffered from a short bowel syndrome due to extensive ileal resection for mesenteric vein thrombosis. Repeated attempts were made at the removal of ductal calculi via ERCP, resulting in partial success. Finally, a percutaneous trans-hepatic approach, allowed stone extraction and balloon dilatation of the bile duct strictures. The patient responded well to this treatment re- 
mained asymptomatic 3 years after the procedure.

\section{Surgical Management}

$5 \%-30 \%$ of patients with PB develop biliary obstruction. Bernon et al. [21] report on a patient with $\mathrm{PB}$ that was successfully managed with an intrahepatic segment 3 bypasses. The latter provides a definitive treatment of bile duct decompression and stone removal in a single procedure in appropriately selected patients.

\section{Conclusion}

$\mathrm{PB}$ is a rare complication of extra-portal venous obstruction associated with $\mathrm{PH}$ and extrahepatic PVT. Most patients remain asymptomatic, but some may present with features of cholangitis. Ischemic injury to the biliary tree is the postulated cause. Extrinsic compression of the common duct by dilated venous collaterals together with pericholedochal fibrosis from an inflammatory process can cause portal thrombosis, which may lead to biliary stricture and dilatation of the proximal biliary tree. These strictures set the scene for formation biliary stones and cholangitis. Imaging plays a vital role in the diagnosis and management of $\mathrm{PB}$. A personal series of imaging of PB is presented. The causation, the course of disease and management particularly minimally invasive procedures is discussed. Imaging is the cornerstone of accurate diagnosis and directs further management. An illustrative atlas is provided supported by patients from our intuition seen over 10 - 15 years. See images with detailed explanations provided. These images are examples of personal series collected over years.

\section{Consent}

Retrospective study, archives from several hospitals, personal series. No consent is required as no direct patient data is published.

Ethical approval is not required, retrospective study.

\section{Contribution of Authors}

Dr. Ali Nawaz Khan: Wrote, designed the study and the images are from personal archives;

Dr. Ken Uzoka: Wrote part of the study and contributed images and provided legends to the images;

Dr. Sumaira Macdonald: Contributed images, and revised the paper study retrieved all relevant documents pertaining to the paper;

Dr. Abeeku Afedzi Hammond: Worked on the images, labelled the images and revised the paper;

Mr. Anthony Kodzo-Grey Venyo: Read the paper and also made changes to the original drafted paper.

\section{References}

[1] Sarin, S.K., Bhatia, V. and Makwane, U. (1992) Portal Biliopathy in Extra Hepatic 
Portal Vein Obstruction. Indian Journal of Gastroenterology, 11, A82.

[2] Chandra, R., Kapoor, D., Tharakan, A., et al. (2001) Portal Biliopathy. Journal of Gastroenterology and Hepatology, 16, 1086-1092. https://doi.org/10.1046/j.1440-1746.2001.02562.x

[3] Fraser, J. and Broun, A.K. (1944) A Clinical Syndrome Associated with a Rare Anomaly of Vena Portal System. Surgery, Gynecology \& Obstetrics, 78, 520-524.

[4] Chevallier, P., Denys, A., Novellas, S., Schmidt, S., Schnyder, P. and Bruneton, J.N. (2006) Magnetic Resonance Cholangiography Features of Biliary Abnormalities Due to Cavernous Transformation of the Portal Vein. Clinical Imaging, 30, 190-194. https://doi.org/10.1016/j.clinimag.2005.12.028

[5] Shin, S.M., Kim, S., Lee, J.W., Kim, C.W., Lee, T.H., Lee, S.H. and Kim, G.H. (2007) Biliary Abnormalities Associated with Portal Biliopathy: Evaluation on MR Cholangiography. American Journal of Roentgenology, 188, 341-347. https://doi.org/10.2214/AJR.05.1649

[6] Baskan, O., Erol, C. and Sahingoz, Y. (2016) Portal Biliopathy, Magnetic Resonance Imaging and Magnetic Resonance Cholangiopancreatography Findings: A Case Series. Gastroenterology Report, 4, 68-72.

[7] Arrivé, L., Ruiz, A., El Mouhadi, S., Azizi, L., Monnier-Cholley, L. and Menu, Y. (2013) MRI of Cholangitis: Traps and Tips. Diagnostic and Interventional Imaging, 94, 75-770. https://doi.org/10.1016/j.diii.2013.03.006

[8] Ozkavukcu, E., Erden, A. and Erden I., (2008) Imaging Features of Portal Biliopathy: Frequency of Involvement Patterns with Emphasis on MRCP. European Journal of Radiology, 71, 129-134. https://doi.org/10.1016/j.ejrad.2008.03.007

[9] Aguilar-Olivos, N.E., De León-Monterroso, J.L., Avila-Escobedo, L. and López-Méndez, E. (2014) Therapeutic Options for Portal Hypertensive Biliopathy: Case Series and Literature Review. Cirugía y Cirujanos, 82, 680-685. [In Spanish]

[10] Matsuo, M., Kanematsu, M., Nishigaki, Y., Kondo, H., Goshima, S., Maeda, S., Tomita, E. and Hoshi, H. (2002) Pseudothrombosis with T2-Weighted Fast Spin-Echo MR Images Caused by Static Portal Venous Flow in Severe Cirrhosis. Journal of Magnetic Resonance Imaging, 15, 199-202. https://doi.org/10.1002/jmri.10048

[11] Nunoi, H., Hirooka, M., Ochi, H., Koizumi, Y., Tokumoto, Y., Abe, M., Tada, F., Ikeda, Y., Matsuura, B., Tanaka, H., Tsuda, T., Mochizuki, T. and Hiasa, Y. (2013) Portal Biliopathy Diagnosed using Color Doppler and Contrast-Enhanced Ultrasound. Internal Medicine, 15, 1055-1059.

[12] Harmanci, O. and Bayraktar, Y. (2012) How Can Portal Vein Cavernous Transformation Cause Chronic Incomplete Biliary Obstruction? World Journal of Gastroenterology, 18, 3375-3378. https://doi.org/10.3748/wjg.v18.i26.3375

[13] Colle, I., Van Vlierberghe, H., Pattyn, P., Troisi, R., Vogelaers, D., de Hemptinne, B. and De Vos, M. (2003) Cholestasis as Presenting Symptom of Portal Cavernoma. Hepatology Research, 25, 32-37.

[14] El-Matary, W., Roberts, E.A., Kim, P., Temple, M., Cutz, E. and Ling, S.C. (2008) Portal Hypertensive Biliopathy: A Rare Cause of Childhood Cholestasis. European Journal of Pediatrics, 167, 1339-1342. https://doi.org/10.1007/s00431-008-0675-4

[15] Mensier, A., Bounoua, F., Beretvas, G., Mosoi, S. and Kissing, D. (2013) Gastric Ulcers Causing Acute Pancreatitis and Portal Biliopathy: What's the Link? Journal of the Pancreas, 14, 646-648.

[16] Oluyemi, A. and Amole, A. (2012) Portal Hypertensive Duodenopathy Manifesting as Kissing Duodenal Ulcers in a Nigerian with Alcoholic Cirrhosis: A Case Report 
and Brief Review of the Literature. Case Reports in Medicine, 2012, Article ID: 618729.

[17] Khan, M.R., Tariq, J. and Raza, R.M.S. (2012) Portal Hypertensive Biliopathy: Review of Pathophysiology and Management. Tropical Gastroenterology, 33, 173-178. https://doi.org/10.7869/tg.2012.44

[18] Chaudhary, A., Dhar, P., Sarin, S.K., Sachdev, A., Agarwal, A.K., Vij, J.C. and Broor, S.L. (1999) Bile Duct Obstruction Due to Portal Biliopathy in Extrahepatic Portal Hypertension: Surgical Management. British Journal of Surgery, 85, 326-329.

[19] Vleggaar, F.P., Van Buuren, H.R. and Laméris, J.S. (1999) Bile Duct Lesions in Portal Vein Thrombosis. Nederlands Tijdschrift Voor Geneeskunde, 143, 2057-2062. (In Dutch)

[20] Perego, P., Cozzi, G. and Bertolini, A. (2003) Portal Biliopathy. Surgical Endoscopy, 17, 351-352. https://doi.org/10.1007/s00464-002-4224-3

[21] Bernon, M.M., Sonderup, M.W., Chinnery, G.E., Bornman, P.C. and Krige, J.E. (2014) Single-Stage Definitive Surgical Treatment for Portal Biliopathy. South African Journal of Surgery, 52, 57-60. https://doi.org/10.7196/sajs.2062 


\section{Supplementary Sheets}

\section{Case 1: Portal biliopathy associated with PVT}
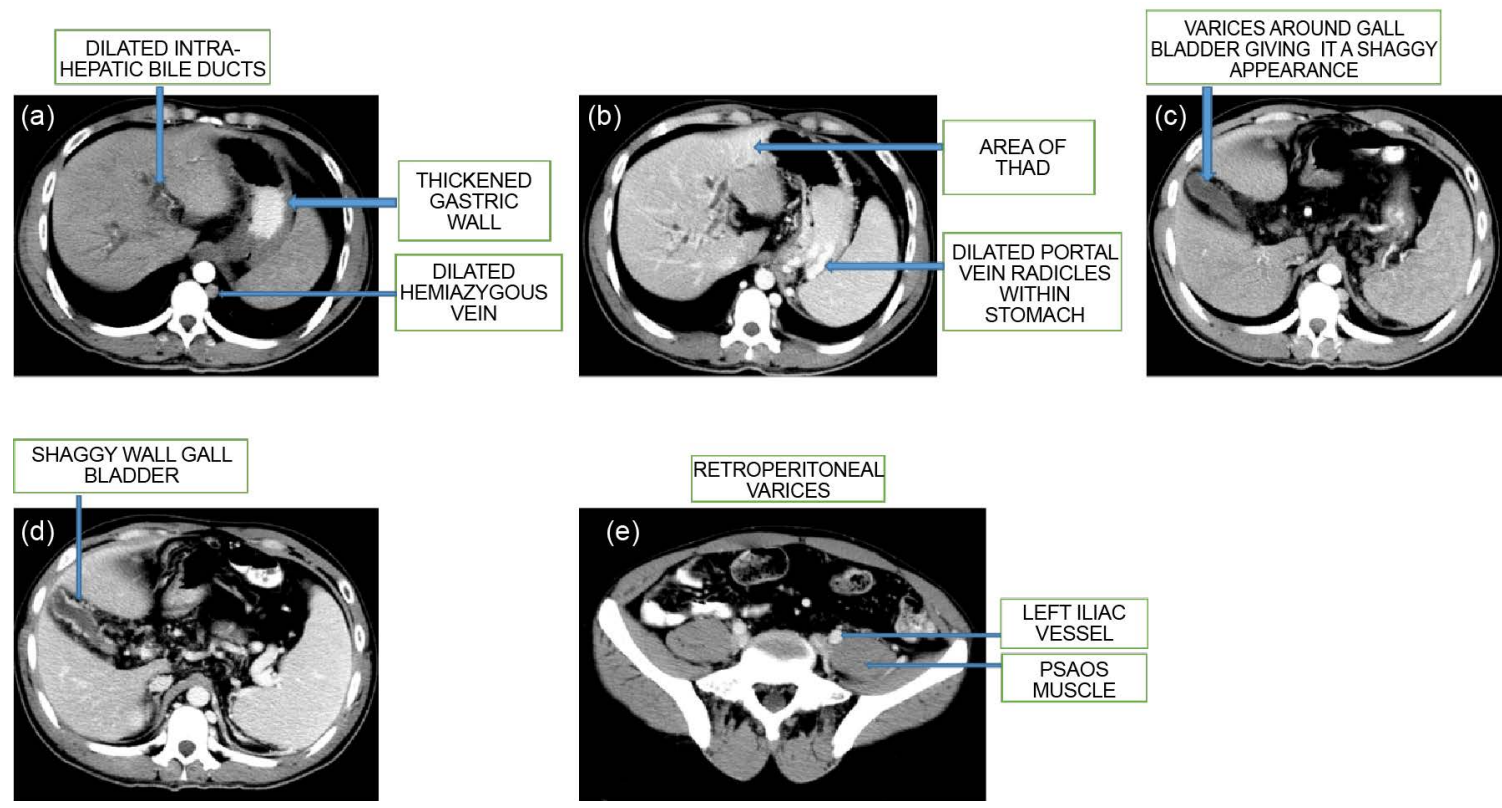

Figure 1. Early arterial phase Axial CT scan through the liver on a patient with known PVT; note the portal hypertensive gastropathy (thickened gastric wall), dilated hemiazygos vein and dilated intrahepatic bile ducts as shown in A. The patient illustrated in Figure 1 survived only 11 months and unfortunately passed away following a massive haematemesis before he could reach the hospital. Despite the sad outcome the case illustrate the findings associated with PB. Also illustrated are other signs such as THAD (Figure 1(b)) not to be confused with a pathology associated with PB. Figure 1(b) shows axial CT scan in the portal venous phase showing dilated portal vein radicles within the stomach lumen, dilated intrahepatic bile ducts, and a dilated hemiazygos vein. There is a THAD (transient attenuation difference) in segment 2 of the liver secondary to a dilated biliary radicle. Arterial and portal phase contrast-enhanced axial CT showed shaggy gall bladder wall due to GB wall varices, minor splenomegaly and retroperitoneal varices around the left psoas sheath secondary to $\mathrm{PH}$ (Figure 1(c), Figure 1(d) and Figure 1(e) respectively).

\section{Case 2: PB-associated with Leber optic atrophy (hereditary optic neuroretinopathy) and alcohol abuse}

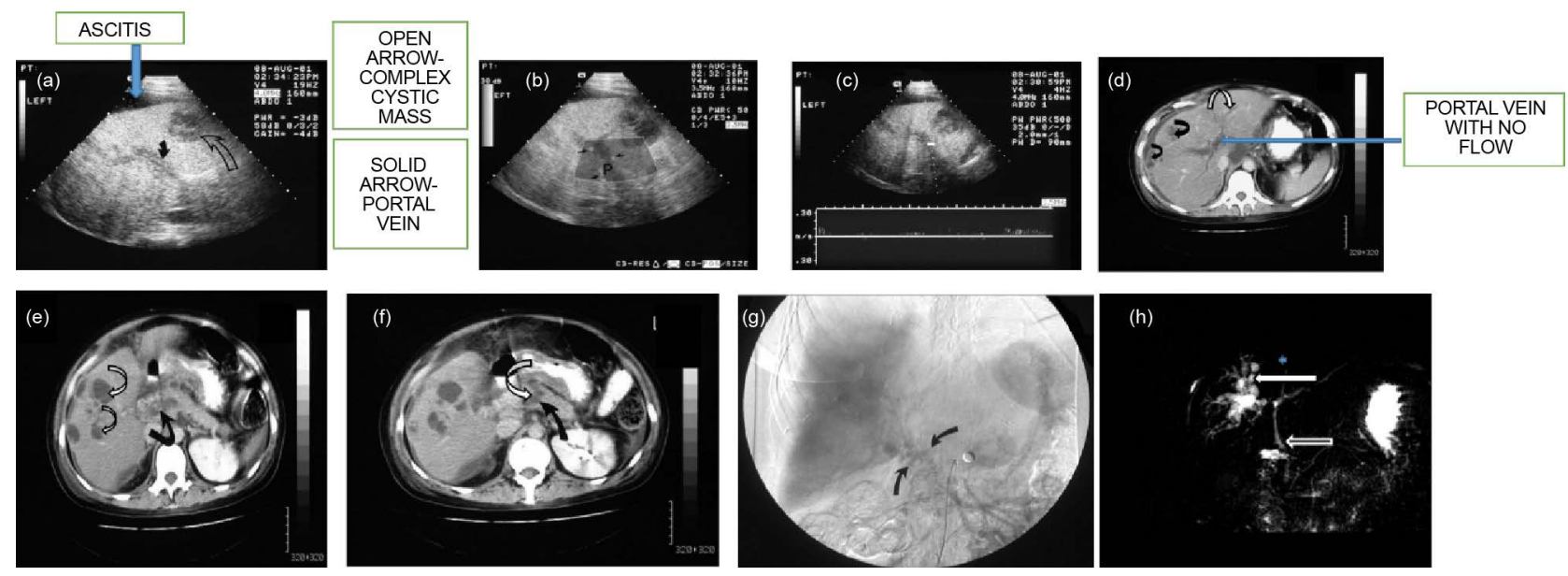

Figure 2. Portal vein thrombosis. A longitudinal oblique sonogram (Figure 2(a)) was obtained through the liver in a 36-year-old woman with a known history of Leber optic atrophy (hereditary optic neuroretinopathy) and alcohol abuse who presented with nonspecific complaints of ill health and vague abdominal pain. Image shows ascites and a bright liver (fatty). The portal vein has 
an echogenic linear structure running the length of the portal vein (solid arrow). A complex cystic mass is present within the liver (open arrow). Figure 2(b) is a power Doppler sonogram of the liver showing blood flow around an intraluminal filling defect in the portal vein $(\mathrm{P})$. Figure $2(\mathrm{c})$ is a spectral Doppler sonogram of the liver, obtained in the same patient as in the previous image, showing the portal vein (cursor), which demonstrates no blood flow. Figure 2(d) is portal venous-phase enhanced axial CT scan showing no flow in the portal vein. Note the multiple low-attenuating masses at the periphery of the right lobe of the liver (solid arrows): Note the dilated intrahepatic ducts (open arrows). There is also a low-attenuating mass (Figure 2(e)) in the termination of the splenic vein (solid arrow). Note the multiple low-attenuating masses (Figure 2(e)) at the periphery of the right lobe of the liver (open arrows). Figure 2(f) is shows a portal venous-phase enhanced axial CT scan, obtained in the same patient, showing an enlarged left gastric vein (open arrow) associated with a low-attenuating mass in the vein. No contrast enhancement is seen in the vein; this finding is suggestive of thrombosis (solid arrow). Digital subtraction portal venous-phase superior mesenteric angiogram Figure 2(g) shows collateral vessels at the porta hepatis but no patent portal vein. Note that the liver is displaced away from the thoracic cage as a result of ascites. This patient had a severe hepatic failure and died within 72 hours after the imaging examination. Postmortem examination showed early cirrhosis, pyogenic fulminant cholangitis, multiple liver abscesses, and portal vein and splenic and left gastric vein thrombosis. Figure 2(h) is an MRCP showing intrahepatic dilatation of the right lobe bile ducts (solid white arrow) and a long stricture of the common bile duct (open white arrow). The patient illustrated in Figure 2 had multiple pathologies and sadly passed away within a few months following failed attempts at re-canalization of the PV. Illustrated is the value of MRCP as a non-invasive Imaging of $\mathrm{PB}$

\section{Case 3: PB and PVT}
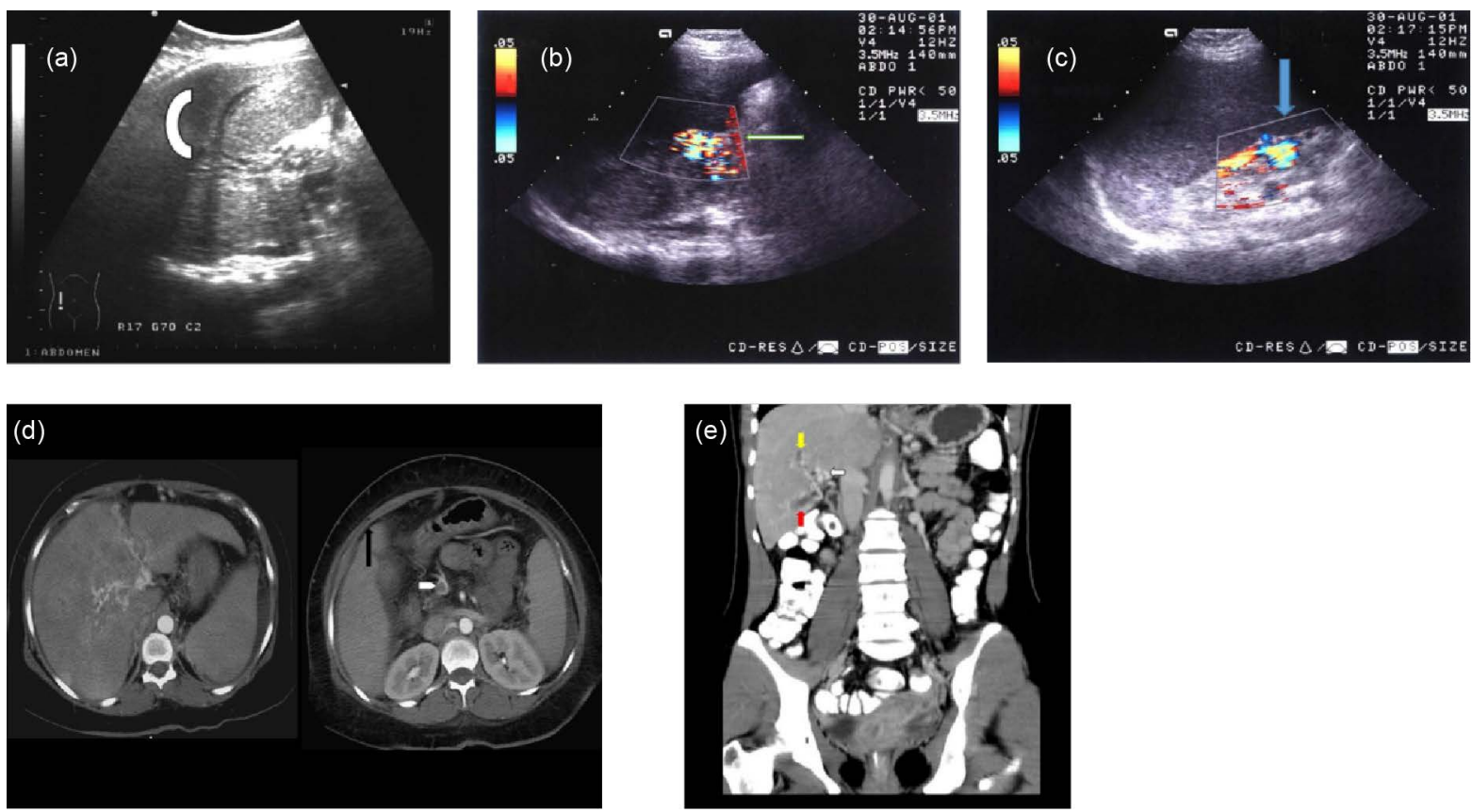

Figure 3. An oblique longitudinal sonogram (Figure 3(a)) was obtained in a 28-year-old woman who was referred for gallbladder ultrasonography. On questioning, she gave a history of an episode of severe pyrexial illness and dehydration during childhood. The image shows several vascular tubular structures at the porta hepatis, which are suggestive of a cavernous transformation (open white arrow bracket showing an area of cavernous transformation). Colour Doppler sonogram (Figure 3(b)) shows flow in the cavernous transformation (arrow). The color Doppler sonogram of the spleen, obtained shows moderate splenomegaly with varices at the splenic hilum (Figure 3(c)). Endoscopic findings confirmed the presence of esophageal varices. The arrow points to the level of splenic hilum showing multiple dilated structures with a flow on Doppler (Figure 3(c)). Figure 3(d) is a Contrast-enhanced axial CT depicts cavernous transformation following portal venous thrombosis. The PVT is extending from the superior mesenteric vein (right image-arrow). Note the small ascites (black arrow).Coronal reconstruction of contrast-enhanced CT (Figure 3(e) depicts cavernous transformation following portal venous thrombosis. Note the gallbladder varices (red arrow), dilated bile duct (yellow arrow) and cavernous transformation (white arrow). The appearances suggest portal biliopathy. The case illustrated to show the value of different modalities and reconstructions to achieve the diagnosis of PB. 


\section{Case 4: PB and PVT}
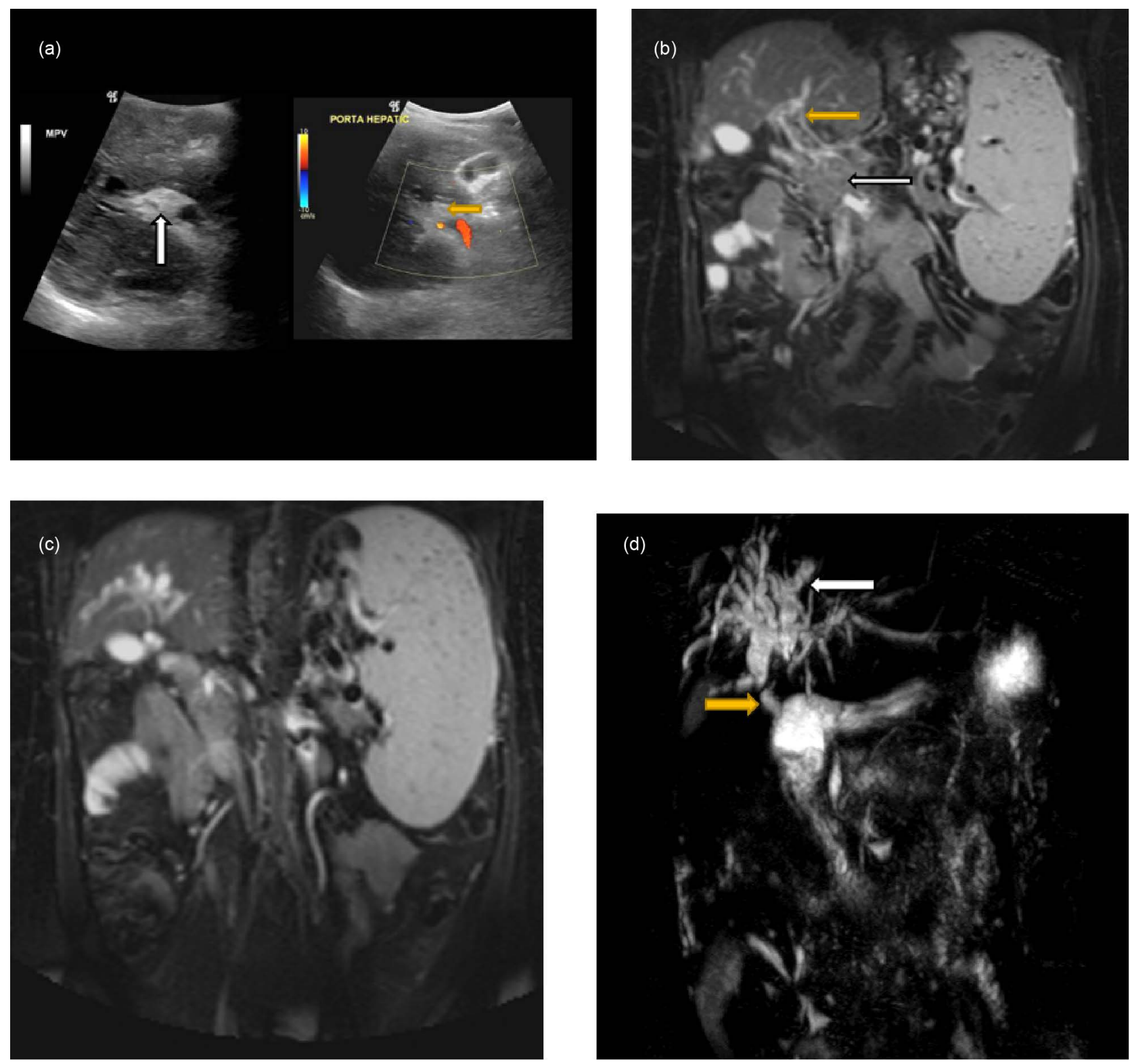

Figure 4. Ultrasound reveals an echogenic partially re-canalized portal vein thrombus (Figure 4(a)). The patient was a 36-year-old woman with idiopathic chronic portal vein thrombosis (white arrow) of 2 years' duration. She presented with cholestatic jaundice. The yellow arrow shows portal vein with no flow. Figure 4(b) and Figure 4(c) is a T1-contrast-enhanced coronal MR images showing thrombus within the main portal vein (white arrow) and a cavernous transformation (yellow arrow) of the portal vein at the porta hepatis. Figure 4(d) is MRCP showing intrahepatic dilatation of the right lobe bile ducts (white arrow) and a long stricture of the common bile duct (yellow arrow). This stricture was successfully dilated by a percutaneous approach with a favourable outcome. 


\section{Case 5: PB-associated with liver transplant}
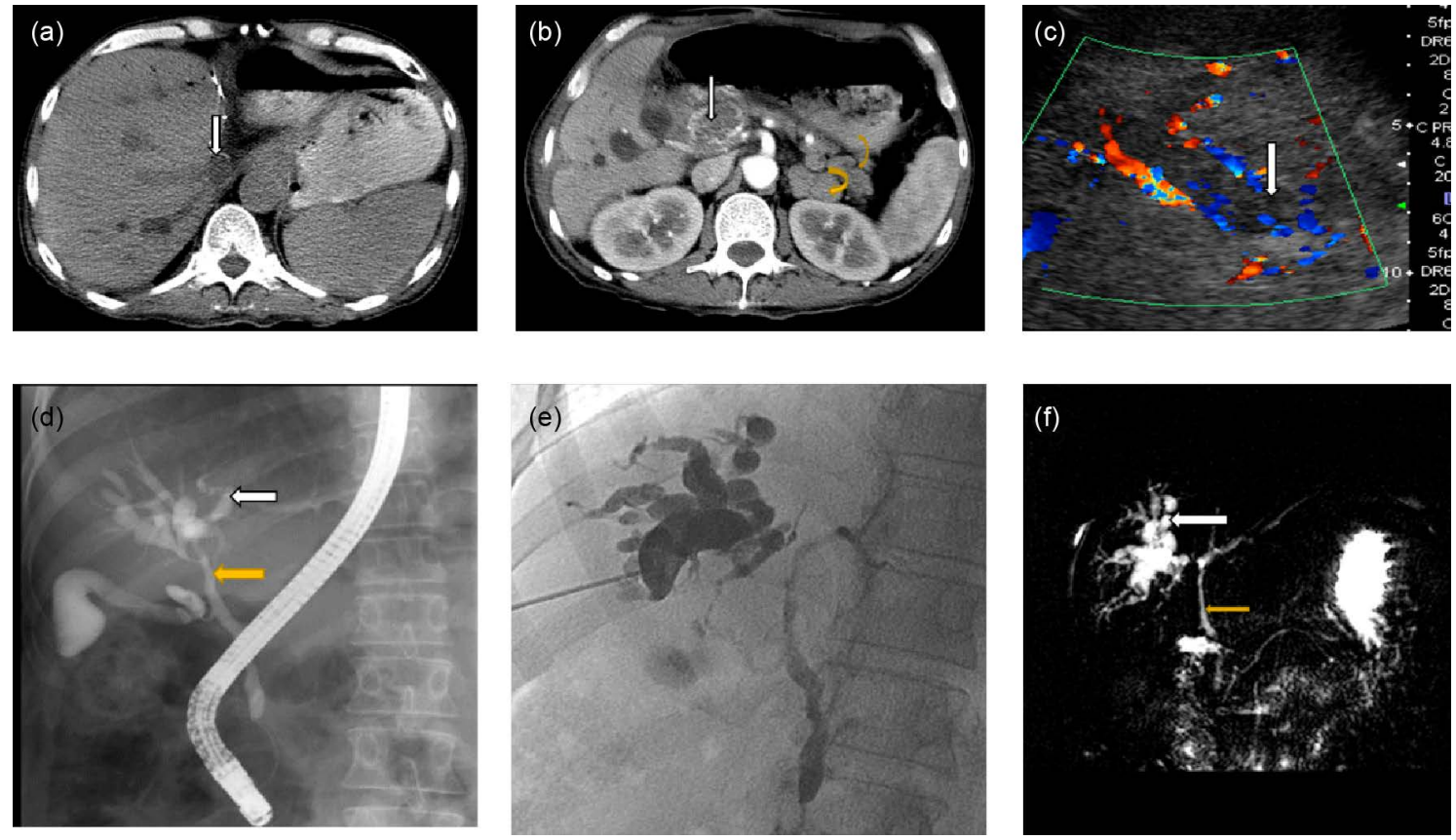

Figure 5. A 53-year-old with LDLT (Live donor liver transplant) presented with cholestatic jaundice, malaise, and weight loss. The axial unenhanced CT scan (Figure 5(a)) shows thrombosis of the IVC (white arrow) and hepatic veins and peripheral small duct dilatation suggestive of PB. Figure 2(a) is an axial arterial phase and early portal venous phase CT scan showing an expanding thrombus of the portal vein (white arrow); note the dilated small peripheral bile duct radicles and varices (yellow arrows) inferior to the body and tail of the pancreas. Figure 5(c) shows a thrombus with the portal vein and periportal varices. Figure 5(d) is an ERCP showing a stricture at the common hepatic duct (yellow arrow) at the porta hepatis and dilatation of the intrahepatic bile ducts (white arrow). Figure 5(e) is a percutaneous transhepatic cholangiogram showing similar findings to the ERCP. Figure 5(e) is MRCP showing similar findings to the ERCP and cholangiography non-invasively. The study shows a dilated intrahepatic ducts (white arrow) and stricture (yellow arrow). Liver transplantation, depending on the type of transplant can be associated with a variety of complications. The PV was successfully dilated by a percutaneous approach with a favourable result.

\section{Case 6: PVT}
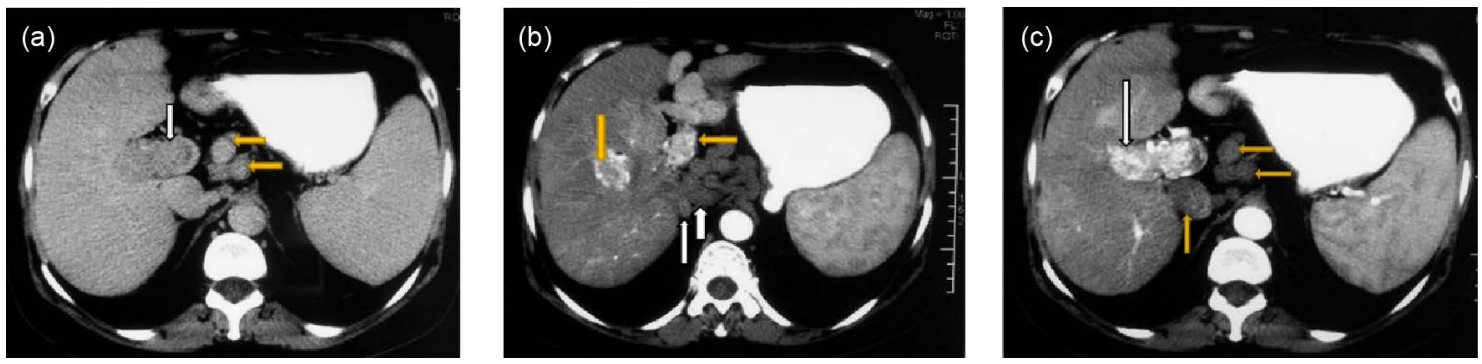

Figure 6. Axial CT scan (Figure 6(a)) on a 21-year-old- woman known to have PVT; note the thrombus within the main portal vein (white arrow), varices at the porta hepatis (yellow arrows) and splenomegaly. Figure 6(b) is an axial CT portal venous phase showing a thrombus within the main portal vein (yellow arrows), large varices (white arrows), periportal contrast enhancement due to periportal varices and uneven contrast enhancement. Figure 6(c) depicts axial CT portal venous phase shows an enhancing thrombus (white arrow) within the main portal vein, and uneven contrast enhancement. The yellow arrows depict varices. These images illustrate a patient that had a pyrexial illness in childhood presented with a mildly raised ALT and a small haematemesis. The patient was offered a portosystemic shunt, which was turned down. Nevertheless, a roadmap has been obtained by imaging, and the patient instructed what to do if symptoms recur. 


\section{Case 7: PB associated with a splenorenal shunt}
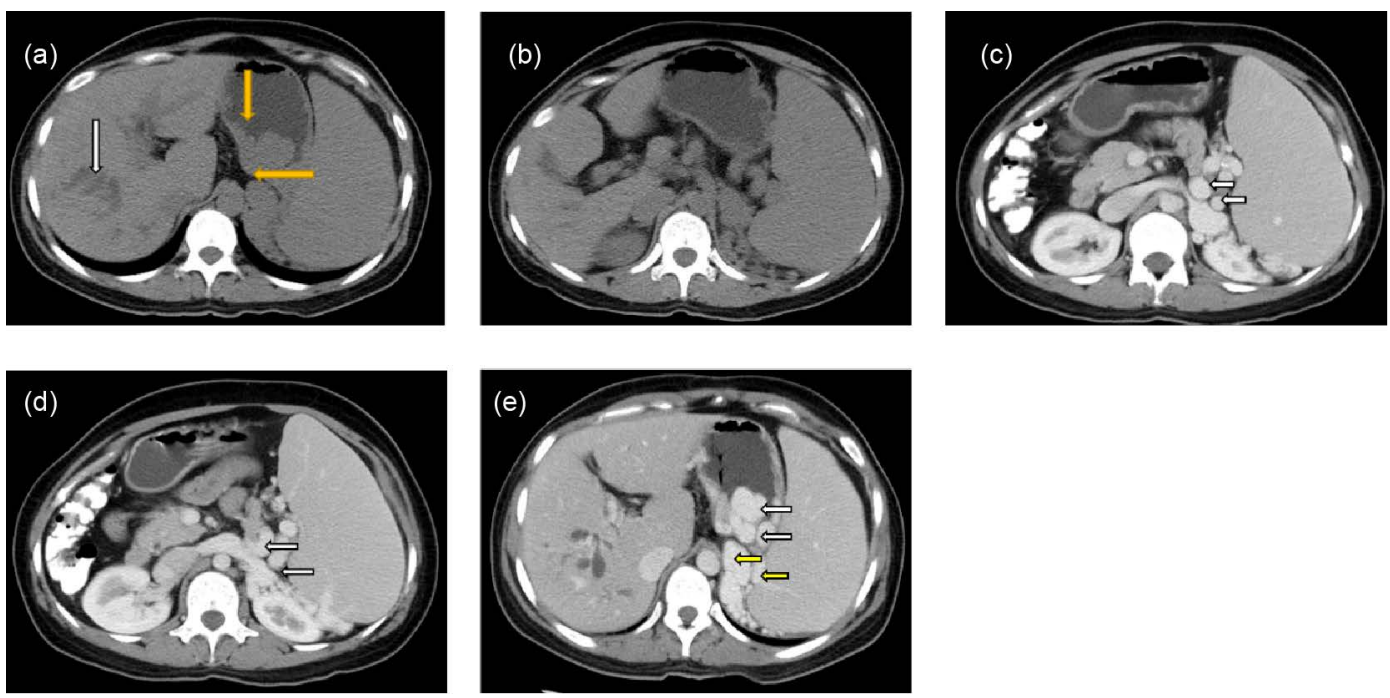

Figure 7. A splenorenal shunt is a complication of PVH and is nature's way of decompression of PVH. The diagnosis is achieved by US/Colour Doppler Imaging but with advances in CT imaging a rapid diagnosis is made with Contrast-enhanced CT. A contrast-enhanced CT scan (Figure 7(a)) showed features of a splenorenal shunt, splenic (yellow arrow) and gastric varices (orange arrow) and dilated intrahepatic bile ducts (white arrow) (PB). The scan Figure 5(c) and Figure 5(d) showed splenomegaly, varices (white arrow) at the above the upper pole of the left kidney, gastric varices, and intrahepatic bile duct dilatation. Figure 5(e) showed features of a splenorenal shunt, splenic (yellow arrow) and gastric varices (white arrow) and dilated intrahepatic bile ducts (black arrow) (PB). This is a 51-year-old-man with portal hypertension and history of portal vein thrombosis.

\section{Case 8: Portal biliopathy associated with Budd-Chiari and PVT}

The diagnosis of PVT associated with a Budd-Chiari syndrome cannot be achieved without imaging, as liver function test abnormalities are non-specific.
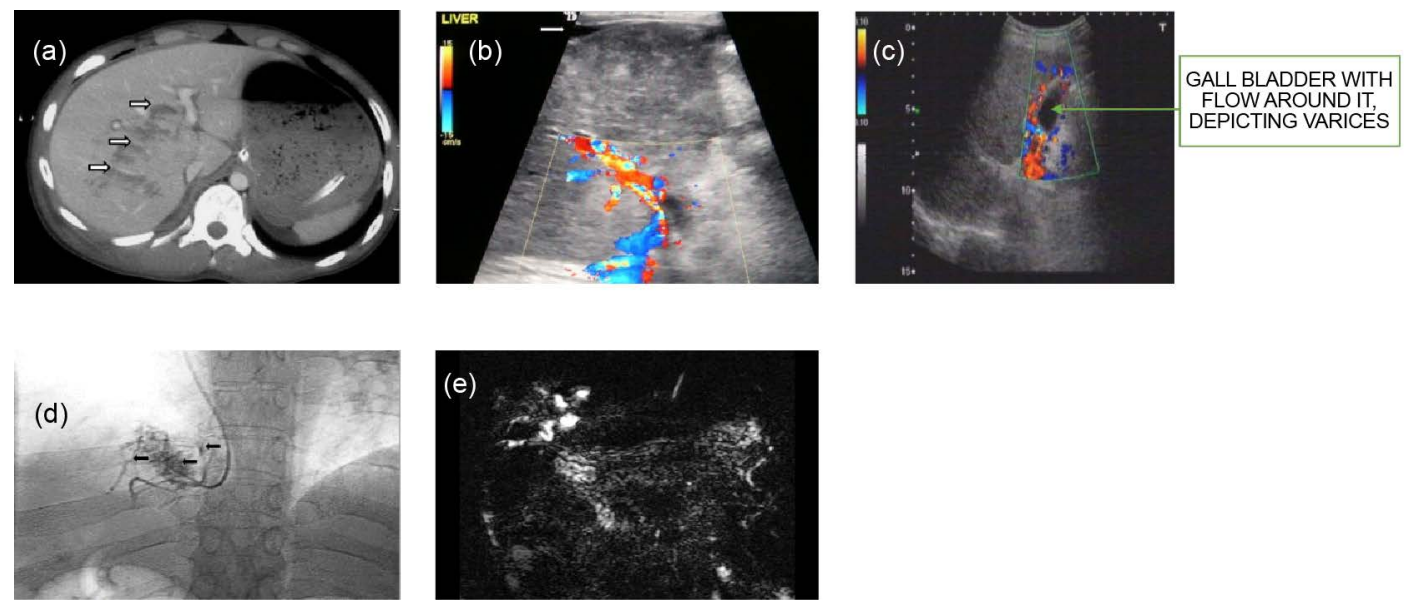

Figure 8. A 28 year-old-woman with known Budd-Chiari syndrome recently with deterioration liver function tests. The axial CT scan performed in portal venous phase (Figure 8(a)) show multiple dilated bile duct radicles (white arrows) due to PB. An oblique ultrasound scan of the liver (Figure 8(b)) shows a cavernous transformation of the portal vein; note the streak of ascites (white arrow). Colour Doppler ultrasound scan of the gallbladder (Figure 8(c)) show gallbladder wall varices. The cavernous transformation of the upper portal vein is also seen. The features described are suggestive of PB. Figure 8(d) depicts wedge hepatic venogram via the right jugular vein showing the characteristic picture of Budd-Chiari syndrome. The arrows show multiple collaterals. Figure 8(e): MRCP on the same patient as in Figures 8(a)-8(c) shows the value of MR imaging in PB. 


\section{Case 9: Portal biliopathy associated with protein C \& S deficiency}

The diagnosis of PB in the elderly particularly in those with Protein C \& S deficiency cannot be achieved by liver function tests, and imaging is vital. Figure 9 shows the value of imaging in such cases.
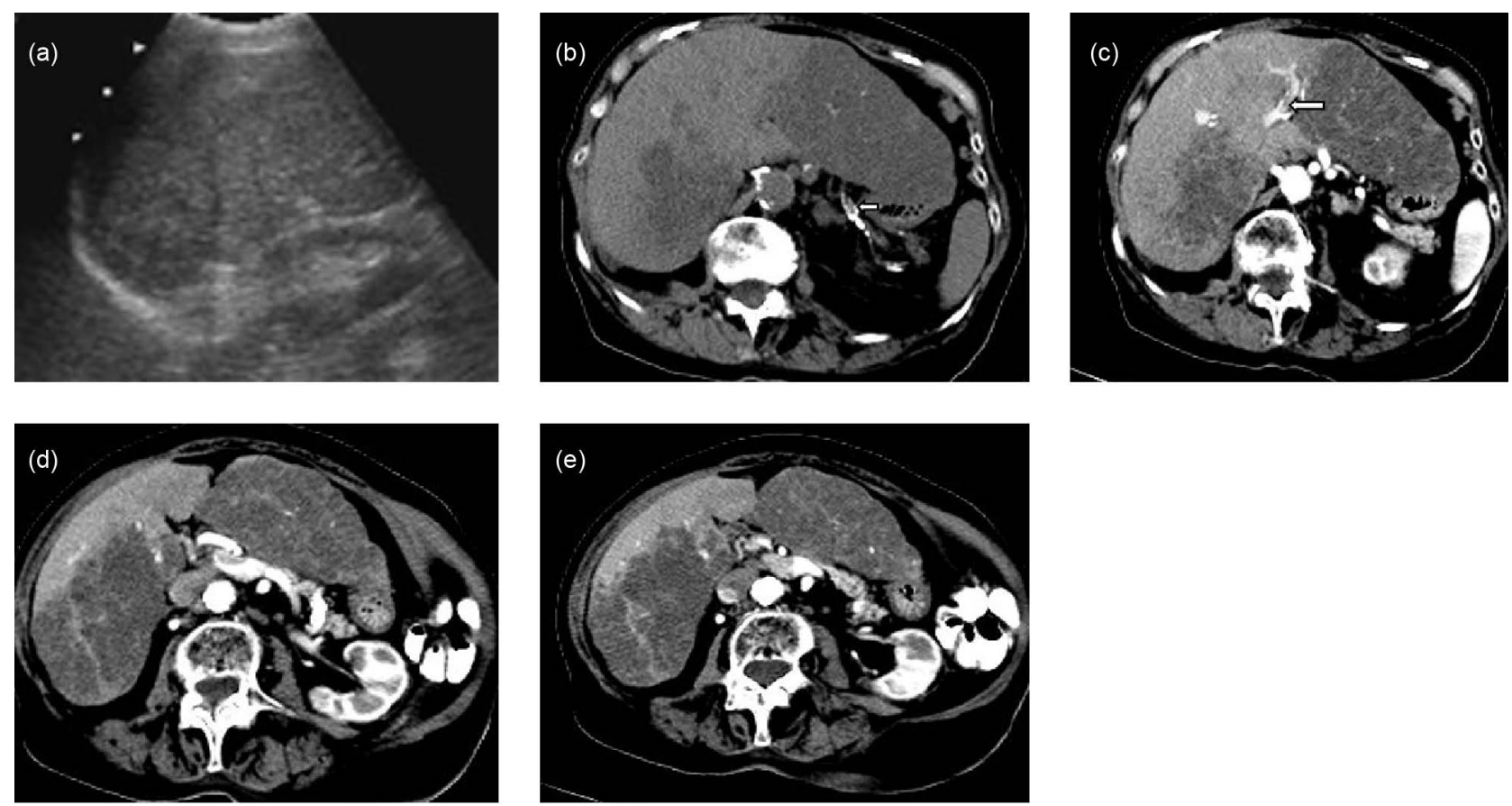

Figure 9. Figure 9(a) is a portable ultrasound scan on 79-year-old-female with a history of protein C deficiency that presented with shortness of breath and deranged liver function tests. The ultrasound scan performed at the bedside showed an abnormal echo pattern in the liver but no features of bile duct obstruction. The patient went on to have a CT scan. The CT scan shows old calcified pulmonary artery thrombosis, PVT, segmental liver infarction and subtle dilated intrahepatic bile duct dilatation suggestive of PB. The patient went on to have a CT scan. The CT scan (Figure 9(b) and Figure 9(e)) shows old calcified pulmonary artery thrombosis (white arrow), PVT, segmental liver infarction and subtle dilated intrahepatic bile duct dilatation suggestive of $\mathrm{PB}$. 